\title{
Additively Manufactured Device for Dynamic Culture of Large Arrays of 3D Tissue Engineered Constructs
}

\author{
Pedro F. Costa, Dietmar W. Hutmacher, * Christina Theodoropoulos, Manuela E. Gomes, \\ Rui L. Reis, and Cédryck Vaquette
}

The ability to test large arrays of cell and biomaterial combinations in 3D environments is still rather limited in the context of tissue engineering and regenerative medicine. This limitation can be generally addressed by employing highly automated and reproducible methodologies. This study reports on the development of a highly versatile and upscalable method based on additive manufacturing for the fabrication of arrays of scaffolds, which are enclosed into individualized perfusion chambers. Devices containing eight scaffolds and their corresponding bioreactor chambers are simultaneously fabricated utilizing a dual extrusion additive manufacturing system. To demonstrate the versatility of the concept, the scaffolds, while enclosed into the device, are subsequently surface-coated with a biomimetic calcium phosphate layer by perfusion with simulated body fluid solution. 96 scaffolds are simultaneously seeded and cultured with human osteoblasts under highly controlled bidirectional perfusion dynamic conditions over 4 weeks. Both coated and noncoated resulting scaffolds show homogeneous cell distribution and high cell viability throughout the 4 weeks culture period and CaP-coated scaffolds result in a significantly increased cell number. The methodology developed in this work exemplifies the applicability of additive manufacturing as a tool for further automation of studies in the field of tissue engineering and regenerative medicine.

\section{Introduction}

Tissue engineering is based on the combination of cells and 3D porous scaffolds to facilitate the replacement and regeneration of damaged tissues. ${ }^{[1]}$ As such, scaffolds should enable both the maintenance of sufficient oxygen diffusion and nutrient supply

Dr. P. F. Costa, Prof. M. E. Gomes, Prof. R. L. Reis 3B's Research Group-Biomaterials Biodegradables and Biomimetics

University of Minho

Avepark-Zona Industrial da Gandra

S. Cláudio do Barco

4806-09 Caldas das Taipas, Guimarães, Portugal

Dr. P. F. Costa, Prof. M. E. Gomes, Prof. R. L. Reis

ICVS/3B's-PT Government Associate Laboratory

Braga/Guimarães, Portugal

Prof. D. W. Hutmacher, Dr. C. Theodoropoulos, Dr. C. Vaquette

Institute of Health and Biomedical Innovation

Queensland University of Technology

60 Musk Avenue, Kelvin Grove, QLD 4059, Australia

E-mail: dietmar.hutmacher@qut.edu.au

DOI: 10.1002/adhm.201400591 in vitro, and provide mechanical support and space maintenance to allow the formation of new tissue and appropriate vascularization in vivo. Further, these scaffolds with a tailored composition/architecture when combined with cells of interest and any necessary biochemical and physical cues, are essential for the successful development of tissue-engineered constructs (TEC) with desirable in vivo properties. ${ }^{[2]}$ This is generally achieved through an extensive and time consuming optimization process.

The utilization of upscalable and modular bioreactors enables the culture of large numbers of constructs under automated and highly controlled conditions simultaneously. This methodology is widely utilized in applications such as high throughput screening (HTS) for screening compounds for drug discovery purposes $^{[3]}$ and has been also proposed for screening 3D structures for tissue engineering applications. ${ }^{[4]}$ However in the majority of these existing devices, several drawbacks remain when screening biomaterials and more specifically $3 \mathrm{D}$ scaffolds. One of the main limitations is the mostly 2D nature of the protocols utilized for investigating cellular response and behavior, which may not necessarily be relevant in $3 \mathrm{D}$ environments. ${ }^{[5]}$ Fluid diffusion and associated nutrient and oxygen supply within a given construct along with cell-material and cell-cell interactions are also crucial parameters, which greatly influence in vitro cellular maturation. These aspects are particularly critical when considering the use of large dimension constructs close to that required in the target application and when the cell culture is performed statically. Flow perfusion bioreactors are traditionally utilized to circumvent these aforementioned issues in scaffolds with significantly larger dimensions. Perfusion improves nutrient supply and distribution and therefore avoids cell death in the most central regions of the scaffolds. ${ }^{[6]}$ However, the lack of versatility in current perfusion bioreactor systems does not permit facile design modifications such as incorporating additional culture chambers and it does not provide the flexibility to utilize scaffolds of various sizes without entirely redesigning the bioreactor system. In some flow perfusion bioreactors, suboptimal fluid flow distribution allows media to flow around the scaffold but does not necessarily perfuse through the construct 
when the scaffold dimension is significantly different to that of the bioreactor chamber. ${ }^{[6]]}$ These design limitations greatly impede the fabrication and testing of large arrays of scaffolds with multiple characteristics (such as architecture, dimensions, shape, etc.) in an upscalable manner.

Additive manufacturing technologies such as fused deposition modeling, offer the possibility to rapidly generate arrays of porous 3D constructs with different shapes and architectures in a highly controlled and reproducible manner. ${ }^{[7]}$ In a previous report, ${ }^{[8]}$ we utilized this technology for fabricating an anatomically relevant device comprising a porous scaffold replica of an ovine tibia around which a bioreactor chamber of similar shape was simultaneously built. We hypothesized that this concept could be further utilized in large-scale cell culture studies while circumventing the aforementioned issues associated with the use of traditional bioreactors. This would enable to reproducibly manufacture arrays of 3D scaffolds and permit their subsequent in vitro culture in a bioreactor chamber in large batches, hence accelerating their characterization. For this purpose, we developed and tested a modular bioreactor device, which was fabricated by dual extrusion fused deposition modeling. This proof of concept study reports on the design, fluid flow optimization, fabrication, and in vitro characterization of the device demonstrating an ability to be further utilized in wide and complex tissue engineering studies (potentially even high throughput strategies).

\section{Results and Discussion}

The present study demonstrates that additive manufacturing can be applied to the fabrication of complex cell culture devices for generating wide arrays of large 3D tissue engineered constructs under highly controlled conditions. As the design and fluid flow optimization are performed at the numerical level and then carried out by the same single automated equipment, the integration of additive manufacturing in large culture/screening studies demonstrates the advantage of generating arrays of 3D scaffolds in a highly reproducible manner while also facilitating possible design modifications (scaffold number, dimension and architecture). The highly 3D nature of the device represents a significant advancement in the field as most large-scale culture systems (in particular, HTS systems) tend to have reduced three-dimensionality and/or in vitro cell culture is performed under static conditions. ${ }^{[9]}$ Also, the automation of the scaffold fabrication along with the bidirectional perfusion enables the systematic assessment of the scaffold performance in a more standardized and highly controlled manner and at an effective cost and speed. It also reduces variations inherent to manual cell culture, such as differences in manual cell seeding efficacy, potential contamination due to frequent media change, as well as variability in volume of media utilized, which can affect oxygen diffusion and therefore result in disparities within samples. Moreover, most bioreactors can test only a few samples at any one time and therefore systematic assessments involving large number of specimens are highly time consuming and labor-intensive. ${ }^{[10]}$ This is in contrast to the technology proposed in the present study, which is unprecedented in the literature as it was capable of testing a large number of highly 3D samples under bidirectional perfusion. Indeed, even in a simplified $4 \times 2$ configuration, 96 $3 \mathrm{D}$ scaffolds contained in 12 separated culture devices were simultaneously cultured demonstrating the upscalability of the developed device.

\subsection{Fluid Flow Modeling}

Fluid flow modeling was used to optimize the design of the individual culture chambers in the developed device. The main requirements considered during this optimization were: (1) homogeneous fluid velocities throughout the entire scaffold; and (2) the elimination of any stagnant fluid regions. Homogeneous fluid distribution ensures a constant and uniform supply of nutrients and oxygen within the construct. When similar culture conditions are achieved throughout the scaffolds, high reproducibility can be achieved. To this end, a number of different configurations of the culture chamber and geometry of the top outlet (Figure 1) were tested.

The geometry of the top outlet was shown to significantly impact on the flow pattern as demonstrated in Figure 1a,b. Indeed an open outlet (Figure 1b) was found to significantly reduce the formation of both low and high velocity zones in the upper portion of the scaffold when compared with a closed outlet configuration (Figure 1a).

Taking into consideration results obtained in a previous study, ${ }^{[11]}$ the design of the chamber was modified to prevent the formation of regions with heterogeneous fluid velocity located at the base of the scaffold. To this end, a porous acrylonitrile butadiene styrene (ABS) structure supporting the PLA scaffold was inserted at the bottom of the chamber. This resulted in positioning the scaffold away from the high velocity regions (represented in yellow and red and highlighted by the black arrows in Figure 1) located in the vicinity of the inlet/outlet. The chambers were chamfered $2 \mathrm{~mm}$ with an angle of $45^{\circ}$ (Figure 1c) to eliminate low fluid velocity regions (white arrows in Figure $1 \mathrm{a}, \mathrm{b})$ as demonstrated in our previous work. ${ }^{[8]}$ This configuration combining the ABS support structure and the chamfered chambers maintained a homogeneous fluid flow velocity throughout the scaffold ranging from $10 \%$ to $15 \%$ of the initial flow rate (Figure 1c). At the inlet of each individual chamber,

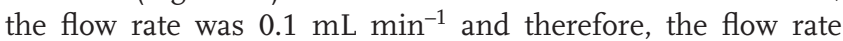
in the scaffold ranged from $0.01-0.015 \mathrm{~mL} \mathrm{~min}^{-1}$ (Figure 1c), which is in agreement with optimal flow rates reported in other studies. ${ }^{[6 a, 12]}$ This optimized design of an individual bioreactor chamber was then utilized to fabricate the upscalable device.

This study demonstrates the proof of principle of this platform into much more complex devices. Our future aim is to generate more elaborate designs comprising multiple conditions within one single device/setup. Similarly to what can be found in the bibliography, these multiple varying conditions may in the future consist of gradients/arrays of multiple reagent concentrations ${ }^{[13]}$ or multiple levels of hydrodynamic shear levels ${ }^{[14]}$ generated by minifluidic channel networks as well as multiple scaffold material compositions by employing multimaterial 3D printers, multiple cellular contents, among other variables. Such 3D cell culture platforms would be highly advantageous for applications in high throughput screening of 


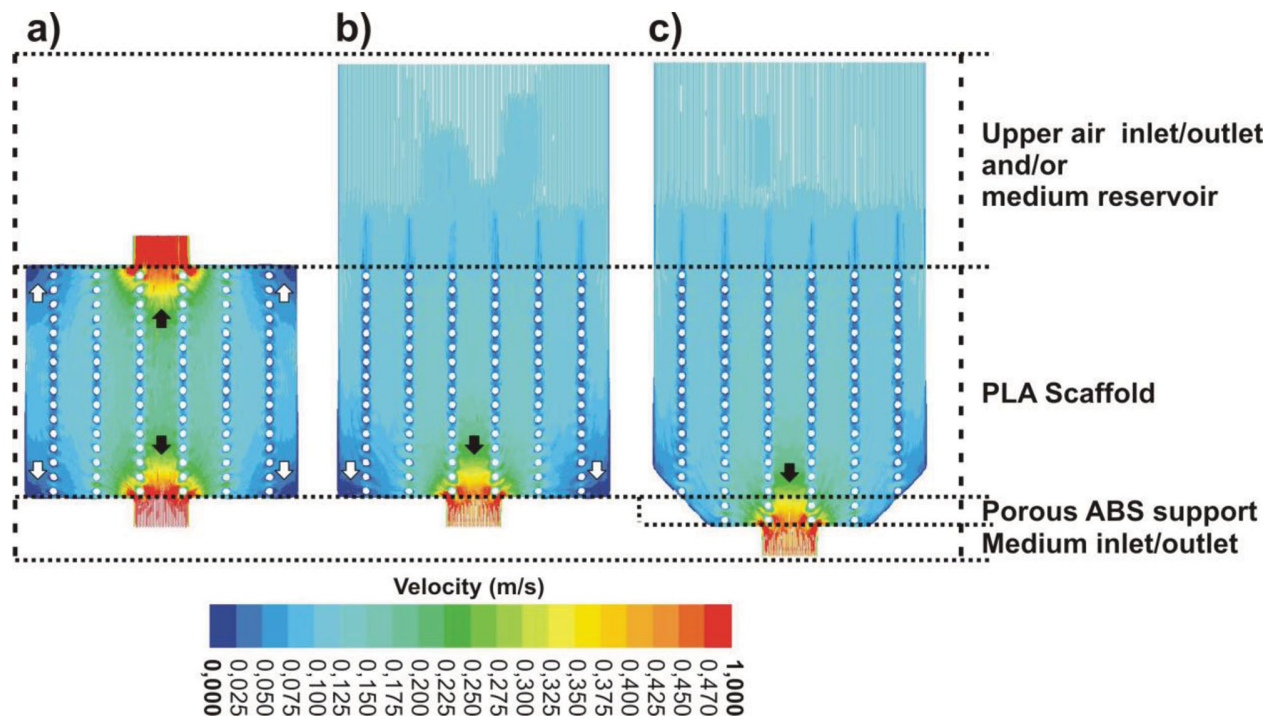

Figure 1. Fluid flow modeling of various device inner architectures. a) Rectangular device with narrow top outlet. b) Rectangular device with wide top outlet. c) $2 \mathrm{~mm}$-chamfered chamber with support structure and wide top outlet. White arrows indicate stagnant zones and black arrows indicate high velocity zones.

drugs in which a large number of cells is needed to perform statistically relevant gene and protein analysis.

\subsection{Design, Fabrication, and Postprocessing of the Device}

3Ds Max software was used to design a multichamber device, which could simultaneously accommodate eight scaffolds (Figure 2a). Figure 2a shows the different elements utilized to create the numerical 3D model. The base of the device integrated a network of 1-mm wide fluidic channels connecting each of the eight culture chambers to one single medium inlet/outlet (Figure 2a1-a2). Based on the fluid flow modeling analysis, the bottom of each chamber was chamfered by $2 \mathrm{~mm}$ along an angle of $45^{\circ}$ (Figure 2a2) onto which, a 1-mm thick porous ABS structure (Figure 2a3) was added to support the PLA scaffold represented in green in Figure 2a4. Each scaffold was surrounded by a $10-\mathrm{mm}$ wide square chamber with $1-\mathrm{mm}$ thick walls and positioned at a distance of $1 \mathrm{~mm}$ from the sides of the scaffolds (Figure 2a5). This chamber was $18 \mathrm{~mm}$ high to create a reservoir for the subsequent cell culture as shown in Figure 2a6. Two pyramidal structures were then placed over each group of four chambers to fully enclose the device. The final model of the device (Figure 2a7) was saved as an STL file, converted to G-Code using RepRap and ReplicatorG software and finally prototyped in a Makerbot dual extrusion fused deposition prototyping machine (Figure 2b1,b2) (Video S1, Supporting Information). The time necessary for fabricating each device was $40 \mathrm{~min}$.

As some open porosity remained within the outer wall of the bioreactor chamber, a surface treatment with an ABS solution was used in order to close these pores ensuring that no leakage would occur during the subsequent cell culture. As a result of this treatment, the outer surfaces of the device became smoother and glossier (Figure 2b5,b6) than nontreated surfaces (Figure $2 \mathrm{~b} 3$ and $2 \mathrm{~b} 4$ ) due to the elimination of the roughness and the interlayer gaps. A more detailed analysis was performed using microCT, which confirmed that most of the porosity initially contained in the bioreactor walls was fully closed or removed by the ABS treatment (Figure 3a1). This analysis also demonstrated that the inner walls and the scaffolds were not affected by the ABS/acetone treatment (Figure 3a2-a4) as shown by the presence of the original interlayer gaps.

The ability to fabricate upscalable culture devices directly from numerically designed 3D models by additive manufacturing offers significant advantages: it reduces costs and human intervention in the fabrication process, while permitting a high degree of control over the size and the architecture of the scaffolds. Generally, the fabrication of culture devices for culture of large arrays of constructs involves the utilization of highly specific technologies and equipment, such as clean room facilities, spin coaters, mask aligners or micromolding, and laser ablation machines, ${ }^{[14 a, 15]}$ which are not necessarily commonly available in a research laboratory and moreover, not as economically viable as additive manufacturing. Furthermore, inherent limitations of these fabrication technologies, such as the need to fabricate one or multiple master molds, result in a time consuming, expensive and largely manual manufacturing process. Devices manufactured in such a way have indeed been largely employed in HTS applications ${ }^{[6 e, 14 a]}$ in order to culture simple-shaped cylindrical scaffolds albeit of smaller dimensions than those developed in the present study. Unlike conventional HTS, where insights are mainly provided at a cellular and hence microscopic level, the device developed in this study shows the ability to provide further knowledge at a more macroscopic/tissue level, hence enabling a more realistic and clinically relevant in vitro assessment. Further, a broad range of materials can be utilized for fabricating scaffolds and therefore contribute to the development of a scaffold materials library. The polymer melt-based deposition process permits the fabrication of objects from a wide array of materials such as aliphatic polyesters, polyurethane, etc. 
a)

1)

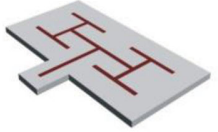

3)

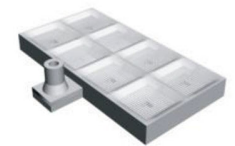

5)

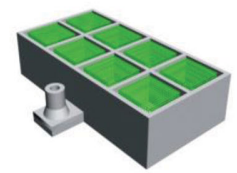

7)

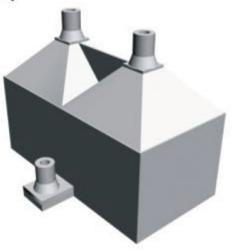

2)

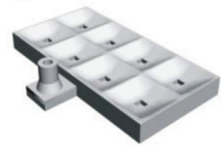

4)

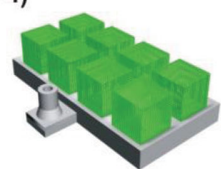

6)

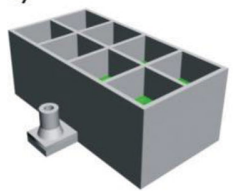

b)
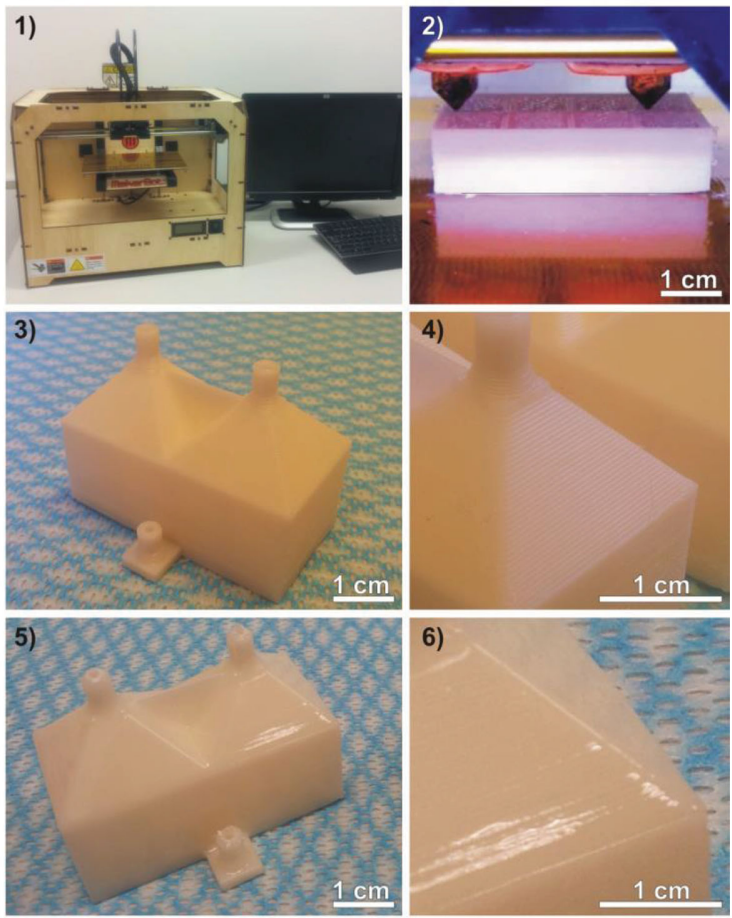

Figure 2. a) Elements of the device to be prototyped. a1) Base of the device consisting of a lower plate designed with a mini channel network for supplying culture medium (red) to the eight culture chambers. a2) The channel network is covered by a perforated layer which defines the chamfered bottom of the culture chambers as well as a lateral inlet/outlet. a3) Porous support structures positioned over the chamber bottoms. a4) Scaffolds positioned over the porous support structures on the bottom of each culture chamber. a5) Walls designed around and in between the scaffolds. a6) Walls extended upwards in order to create an upper reservoir over the scaffolds. a7) Two covers designed on the top part of the device to enclose the culture chambers. The top of each cover possesses an inlet/outlet for air circulation. b) Fabrication and posttreatment of device. b1) Dual extrusion prototyping machine. b2) Close-up view of device being fabricated in the prototyping machine by dual extrusion of ABS (left extruder) and PLA (right extruder). b3,b4) Device after rapid prototyping. b5,b6) Device after posttreatment with ABS/acetone solution.

To further demonstrate the versatility of our strategy, the PLA scaffolds were further coated with a calcium phosphate layer by successively filling the individual culture chambers with sodium hydroxide $(\mathrm{NaOH})$ and hypersaturated simulated body fluid (SBF). Treatment with $\mathrm{NaOH}$ increased the hydrophilicity of the scaffold by increasing the roughness (as shown by the microporosity in Figure $3 \mathrm{~b} 1, \mathrm{~b} 2$ ) and is also known to increase the density of carboxylic acid on the surface of the polymeric struts, which has a significant impact on cell adhesion. ${ }^{[16]}$ The SBF treatment resulted in the deposition of biomimetic calcium phosphate crystals. However, this deposition was not entirely homogenous as agglomerations of CaP spherical particles were found in many locations of the scaffold as shown in Figure 3b3,b4, unlike what was reported in another study utilizing a perfusion system for coating scaffolds of similar architecture. ${ }^{[17]}$ The heterogeneity in the CaP coating probably originated from the relatively short exposition time and the reduced volume of SBF solution utilized, which prevented the formation of a continuous $\mathrm{CaP}$ layer. The $\mathrm{NaOH}$ posttreatment was performed to obtain a single phase of $\mathrm{CaP}$ as a previous study, although performed on a different scaffold, demonstrated that a highly soluble CaP phase (brushite) was removed by the gentle etching, leaving a unique carbonate hydroxylapatite layer. ${ }^{[18]}$ These two treatments $(\mathrm{NaOH}$ etching and $\mathrm{CaP}$ coating) demonstrate that our device also enables the evaluation of functionalized scaffolds.

\subsection{Perfusion Cell Culture}

Another important aspect of the technology we have developed is that it enables the in vitro culture of the scaffold under bidirectional perfusion. The perfusion apparatus was assembled by connecting a syringe to the bottom inlet/outlet of each device using sterile silicone tubing. To preserve the sterility of the device, the top outlets were capped with sterile $0.22 \mu \mathrm{m}$ filters (Figure 4a2). Each silicone tube was then connected to an individual syringe placed onto a multisyringe adapter (Figure 4a1), which was mounted into one single syringe pump. The utilization of a culture setup comprising multiple eight chamber devices connected to individual syringes mounted onto one single syringe pump was chosen in order to show a simple and user-friendly scalability of the culture platform; either by varying the number of device chambers, the number of devices/ syringes and/or the number of syringe pumps and therefore demonstrate versatility and modularity of the technology platform. The devices were then filled with culture medium and the scaffolds incubated overnight to enable protein adsorption. The following day, cell suspensions were loaded into the devices through the silicone tubing and bidirectionally perfused to allow homogeneous cell seeding (Video S2, Supporting Information). Scaffolds were collected after 24 h, 2 and 4 weeks of 
a)
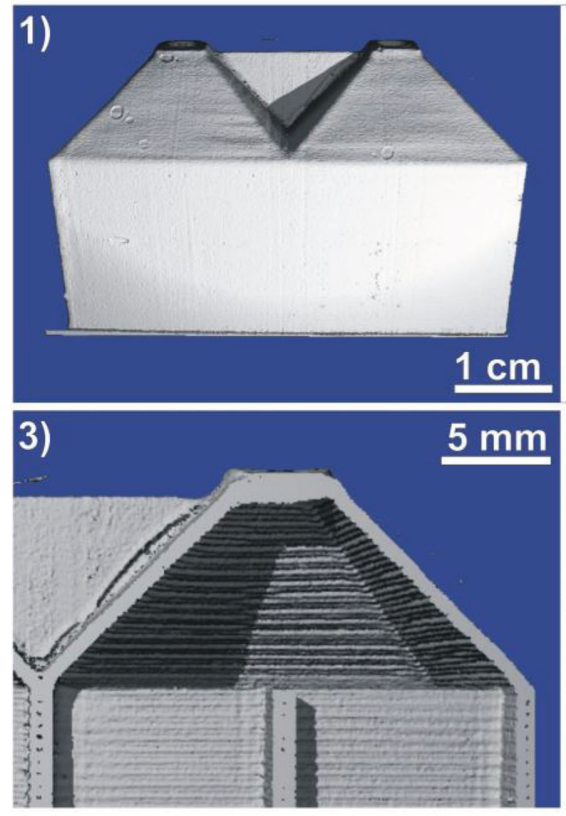

b)
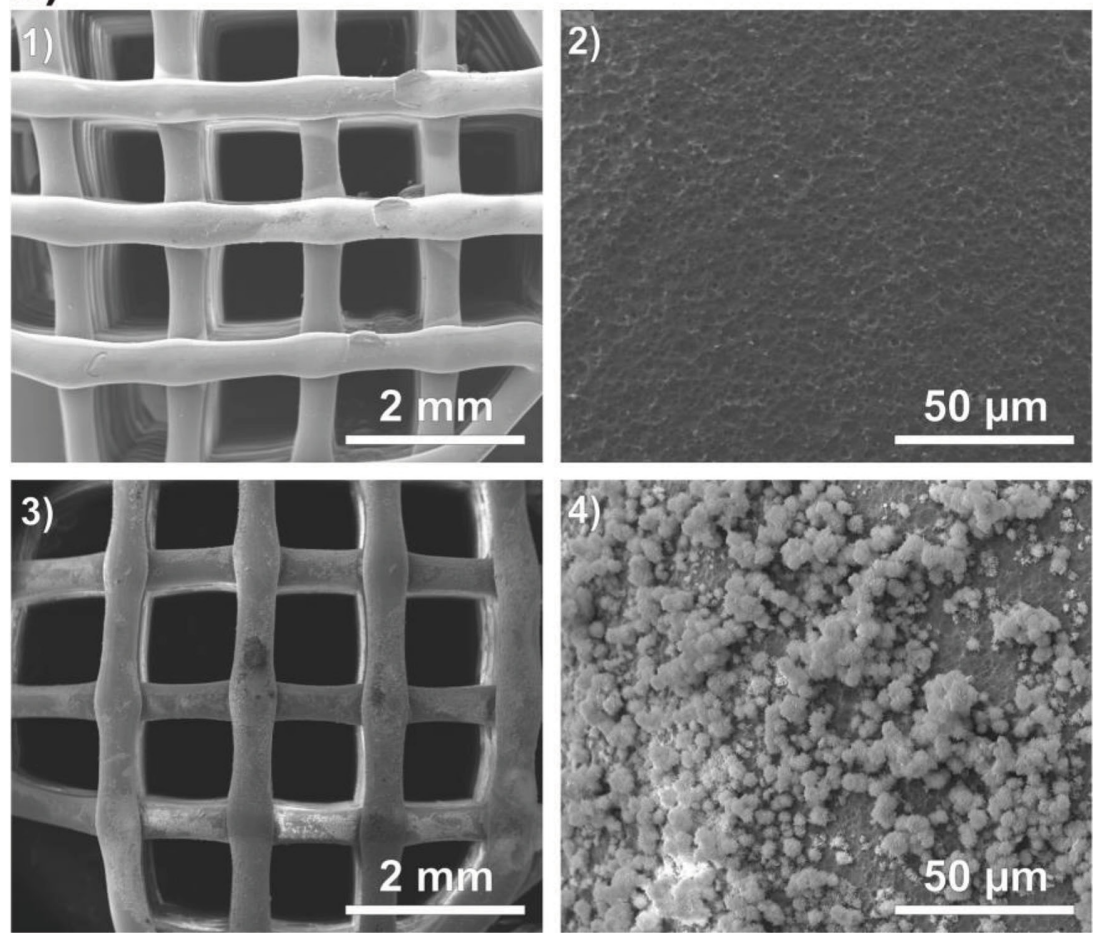

Figure 3. a) Micro-CT reconstruction of device. a1) 3D reconstruction featuring the smooth ABS external wall of the device after posttreatment with an ABS/acetone solution. a2) Crosssection depicting the structure of the device containing the PLA scaffolds. a3) Morphology of the inner surface of the bioreactor chamber which is characteristic of a layer-by-layer deposition suggesting that the ABS-acetone posttreatment did not affect the inner wall of the bioreactor chamber. a4) Close-up view depicting scaffold architecture and the bottom part of device. Arrows indicate sections of mini-fluidic channels. b) SEM images of scaffolds before (b1,b2) and after CaP coating (b3,b4). culture using sterile scissors and tweezers according to the method shown in Figure $4 \mathrm{~b}$ and Video S3 (Supporting Information). DNA quantification revealed that the device supported cell adhesion and proliferation in all types of scaffold as shown in Figure 5a, although these parameters were significantly higher in the CaP-coated specimens. Indeed, the DNA content increased fourfold for the noncoated samples between $24 \mathrm{~h}$ and 4 weeks postseeding, whereas it increased 11-fold for the CaP-coated specimens within the same time period. This finding is supported by several studies, which reported a significant increase in the cell proliferation on CaP-coated scaffolds. ${ }^{[19]}$ This is explained by the release of $\mathrm{Ca}^{2+}$ cations into the culture media from the $\mathrm{CaP}$ coating, which stimulates cell growth. In our previous work, we have demonstrated that such CaP layers deposited over the surface of polymeric scaffolds are able to enhance in vivo bone formation. ${ }^{[20]}$ The scaffolds were stained with 3-(4,5-dimethylthiazol-2-yl)2,5-diphenyltetrazolium bromide (MTT) to assess the cell distribution within the scaffold. Despite low staining efficacy, at the earliest timepoints metabolically active cells had homogeneously infiltrated the scaffolds regardless of the surface treatment (coated and noncoated) as shown in Figure 5b. This shows that the chamber design was effective in homogeneously distributing the cells (highlighted by the white arrows) throughout the scaffold and hence validates the fluid flow simulation analysis. This is also in accordance with a previous study ${ }^{[8]}$ employing a similar seeding methodology, where we have been able to homogeneously seed cells into significantly larger and irregularly shaped scaffolds with no detrimental effect on cellular distribution or viability throughout the entire scaffold architecture. After 4 weeks of culture, the polymeric struts of noncoated scaffolds were covered by a dense layer of single cells while CaP-coated scaffolds were covered by thick sheets of cells (in red) bridging multiple struts (highlighted by black arrows in Figure $5 b)$. Scanning electron microscopy (SEM) confirmed the homogeneous cell distribution in both scaffold groups and showed that the cells gradually covered the polymeric struts and were capable of bridging adjacent struts and partially filling the pores of the scaffold at 4 weeks postseeding as shown in Figure 6 . The distribution and viability of cells in several 
a)
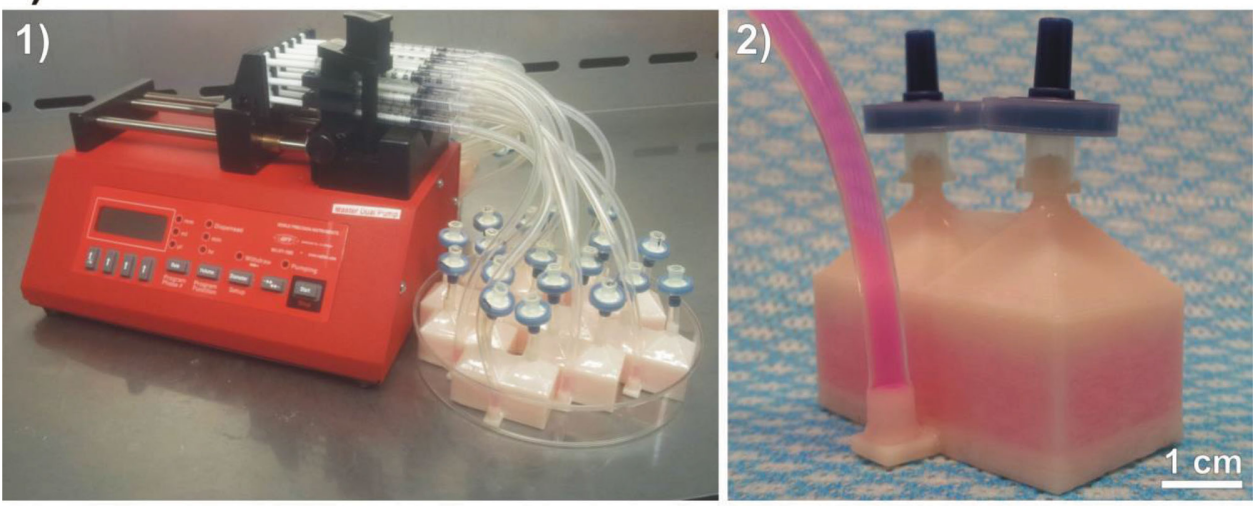

b)

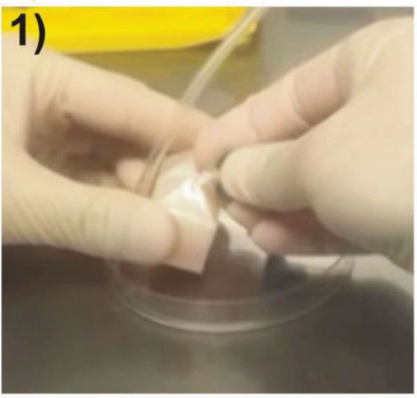

2)
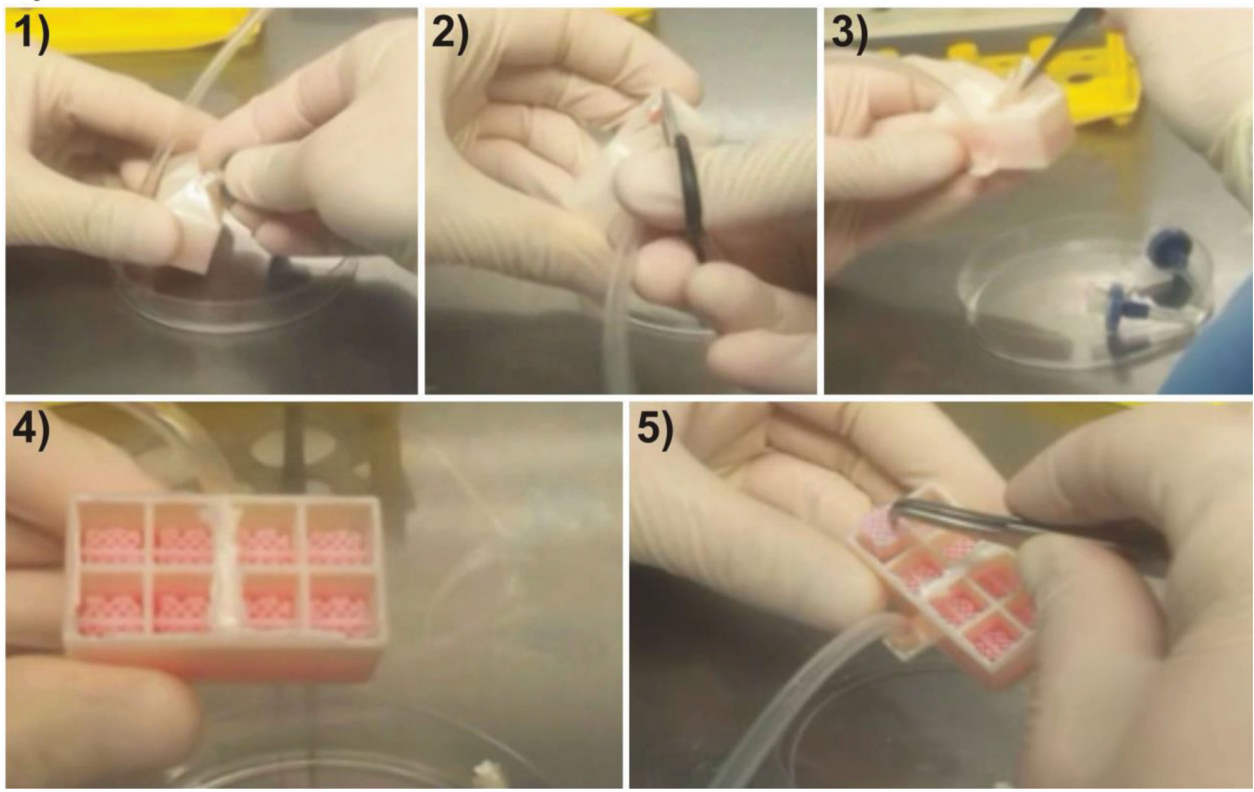

Figure 4. a) Culture system apparatus. a1) Multiple culture devices connected to one single syringe pump for bidirectional perfusion. a2) Device being perfused with culture medium through silicone tubing connected to the lower medium inlet/outlet and with upper air inlets/outlets capped with filters. b) Procedure for retrieving the constructs from the culture device by manually breaking and cutting with the aid of sterile scissors and tweezers.

locations of the scaffolds were also assessed by FDA/PI-staining combined with confocal laser microscopy (Figure 6). Despite the presence of some dead cells (red) the majority of cells were alive (green) at the 4 weeks culture period showing that sufficient nutrient supply was provided throughout the entire scaffold architecture. This was further shown in the semiquantitative analysis in which, both coated and noncoated samples displayed a percentage of live cells above $85 \%$ after 4 weeks of dynamic culture (Table 1).

\section{Conclusion}

A unique dynamic cell culture platform based on applying additive manufacturing has been developed for simultaneous culture of large arrays of scaffolds under highly controlled perfusion culture conditions. The versatility and upscalability of this concept were assessed by manufacturing a device composed of eight scaffolds placed into individualized chambers, which were further modified by alkaline treatment and by simulated body fluid to generate a biomimetic surface coating. The highly scalable nature of the strategy was demonstrated by easily culturing 96 scaffolds in one single experiment. The scaffolds, regardless of the surface treatment, were successfully seeded and cultured with osteoblasts for up to 4 weeks generating constructs with good viability. The utilization of additive manufacturing to build both scaffold and bioreactor in one fabrication step provides a new tool for allowing a higher level of automation of tissue engineering strategies under 3D and highly controlled dynamic environments.

\section{Experimental Section}

Design and Fabrication Concept: Devices targeting large-scale culture applications are required to contain multiple samples for 
www.MaterialsViews.com

a)

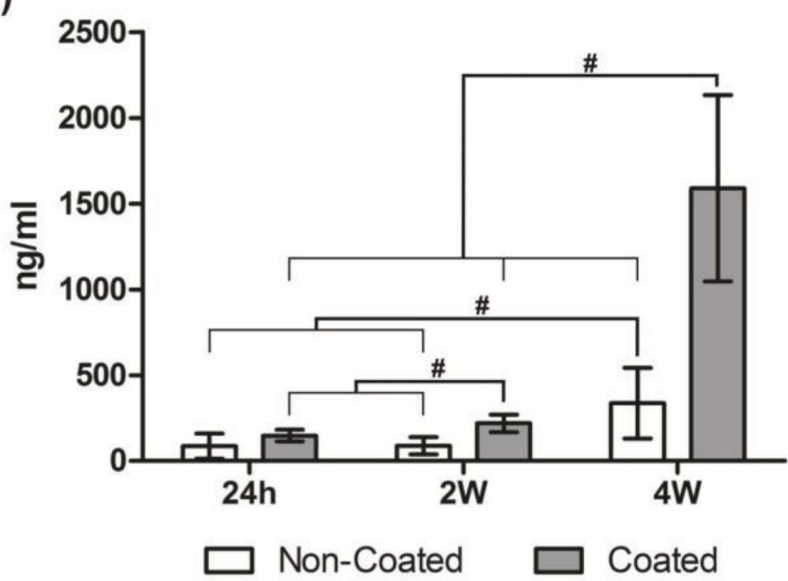

b)
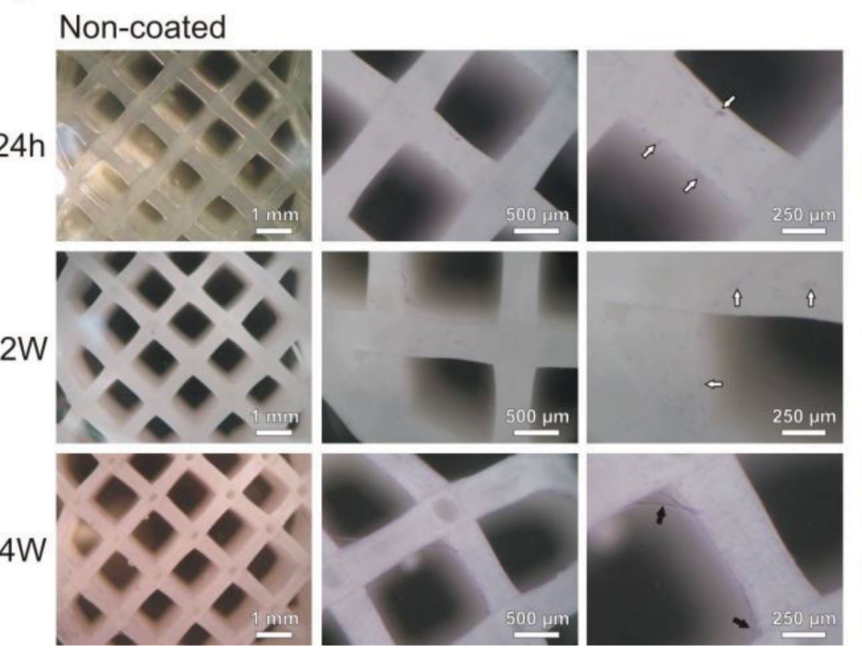

Coated
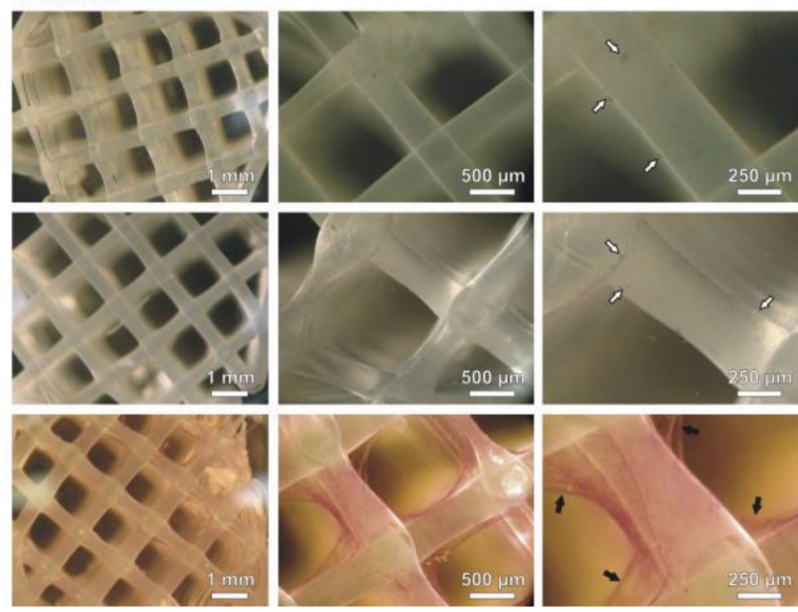

Figure 5. a) DNA content in noncoated and CaP-coated scaffolds after $24 \mathrm{~h}, 2$ weeks and 4 weeks of culture. \# shows statistically different values $(p<0.05)$. b) MTT staining of coated and noncoated samples seeded with osteoblasts after $24 \mathrm{~h}, 2$ and 4 weeks of culture. At the 4 weeks timepoint noncoated scaffolds struts were covered by a dense layer of single cells while CaP-coated scaffolds were covered by thick sheets of cells. White arrows indicate isolated adhered cells while black arrows indicate cellular bridging between struts.

simultaneous testing and handling. This is usually achieved by using versatile test platforms and by process automation. The device utilized in this study was designed to be modular and to allow for upscaling according to the number of scaffolds required. As a proof of concept, a device was designed to simultaneously accommodate eight cubical scaffolds ( $8 \mathrm{~mm}$ width) composed of $400 \mu \mathrm{m}$ diameter struts $1.5 \mathrm{~mm}$ horizontally spaced in a $0-90^{\circ}$ orientation. Device design was performed in 3Ds Max (Autodesk, USA) software and consisted of building individualized bioreactor chambers by placing $1 \mathrm{~mm}$ thick walls around each of the eight scaffolds, which were organized in a rectangular $2 \times 4$ configuration. The walls were positioned $1 \mathrm{~mm}$ from the scaffolds and were $10 \mathrm{~mm}$ taller than the scaffolds in order to create a reservoir for the culture medium. The top of every four chambers (in a square configuration) was covered by a pyramidal structure ending in a $2 \mathrm{~mm}$ diameter outlet used for attaching sterile filters during subsequent cell culture. A symmetrical minifluidic channel network connected to a $2 \mathrm{~mm}$ diameter inlet/outlet was designed for homogenously distributing the culture medium through the various individual chambers.

Fluid Flow Modeling: Computational fluid dynamics was used to simulate the flow pattern of the media in various device architectures. The studied architectures differed in two main aspects: the use of either rectangular or chamfered chamber bottom corners and the use of either wide or narrow top inlets/outlets. Given the high complexity of the device, a simplified model consisting of 2D sections was first created in Design Modeler 13.0 software (Ansys, USA). The profile corresponding to the section of an $8 \mathrm{~mm}$ wide cubic porous scaffold surrounded by a $10 \mathrm{~mm}$ wide bioreactor chamber was considered. The thickness of the simplified section was $0.1 \mathrm{~mm}$, while a converged free mesh was generated using automatic element selection. By applying a free mesh with maximum face size of $0.2 \mathrm{~mm}$, it was possible to reach a detailed and accurate solution with mesh independence. The fluid flow velocity profiles were calculated using Fluent 13.0 software within Ansys Workbench 13.0 platform (Ansys, USA). The pressure at the device's outlets was assumed to be zero and the scaffold and the bioreactor chamber were considered rigid and impermeable. We assumed that the viscosity and the density of the culture media at $37^{\circ} \mathrm{C}$ were $\eta=1.45 \times 10^{-3}$ Pa.s and $\rho=1000 \mathrm{~kg} \mathrm{~m}^{-3}$, respectively.

Conversion, Slicing, and Prototyping of 3D Models: The device design displaying the most homogeneous fluid flow pattern was selected for further experiments. The device's 3D model was then sliced and converted to a G-Code file. The volumes corresponding to the eight cubes to be converted to porous scaffolds were processed using the open source software Reprap (Online Reprap Community). The cubes were sliced into $0.27 \mathrm{~mm}$ layers composed of deposition path lines with 

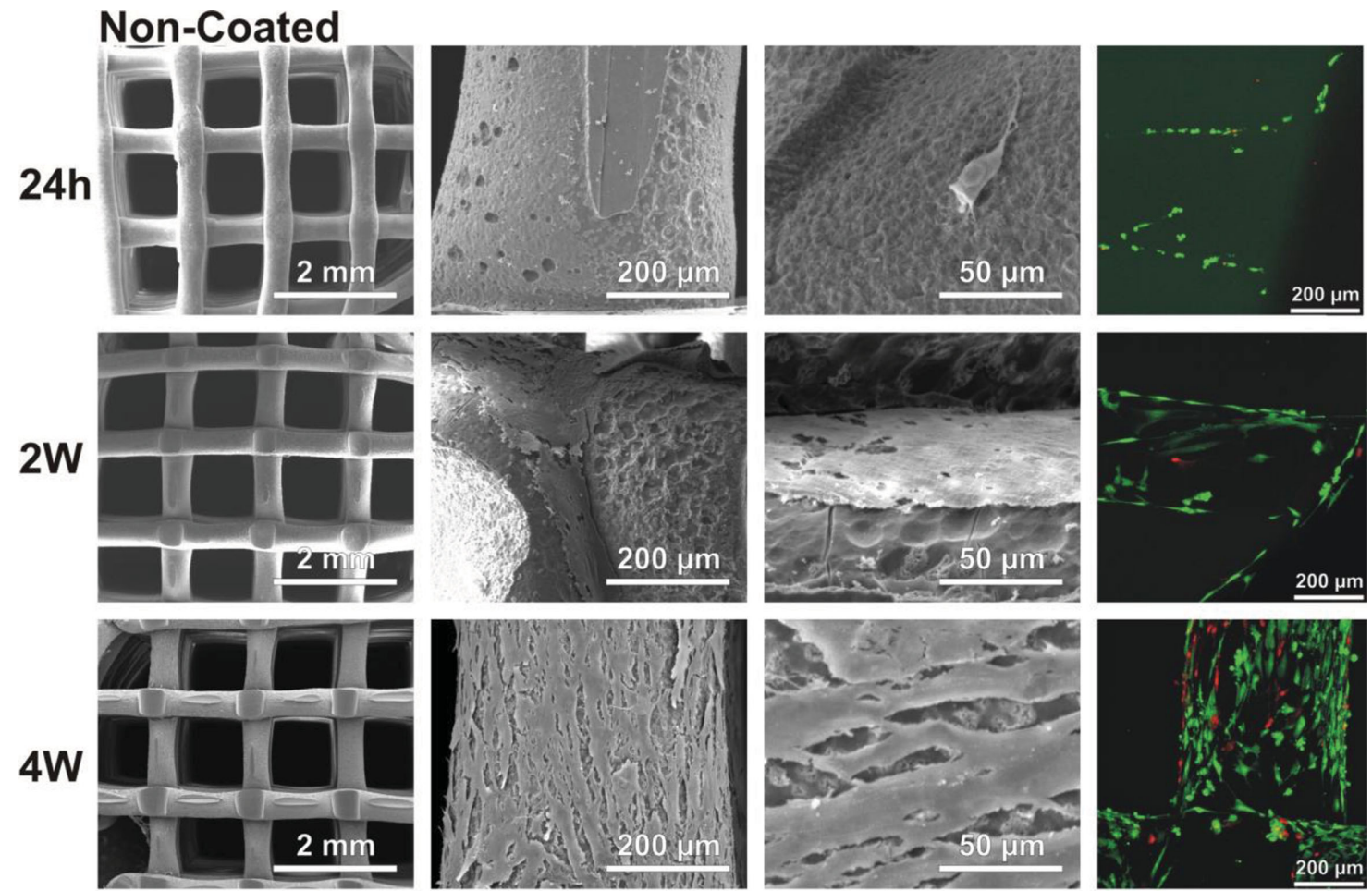

\section{Coated}
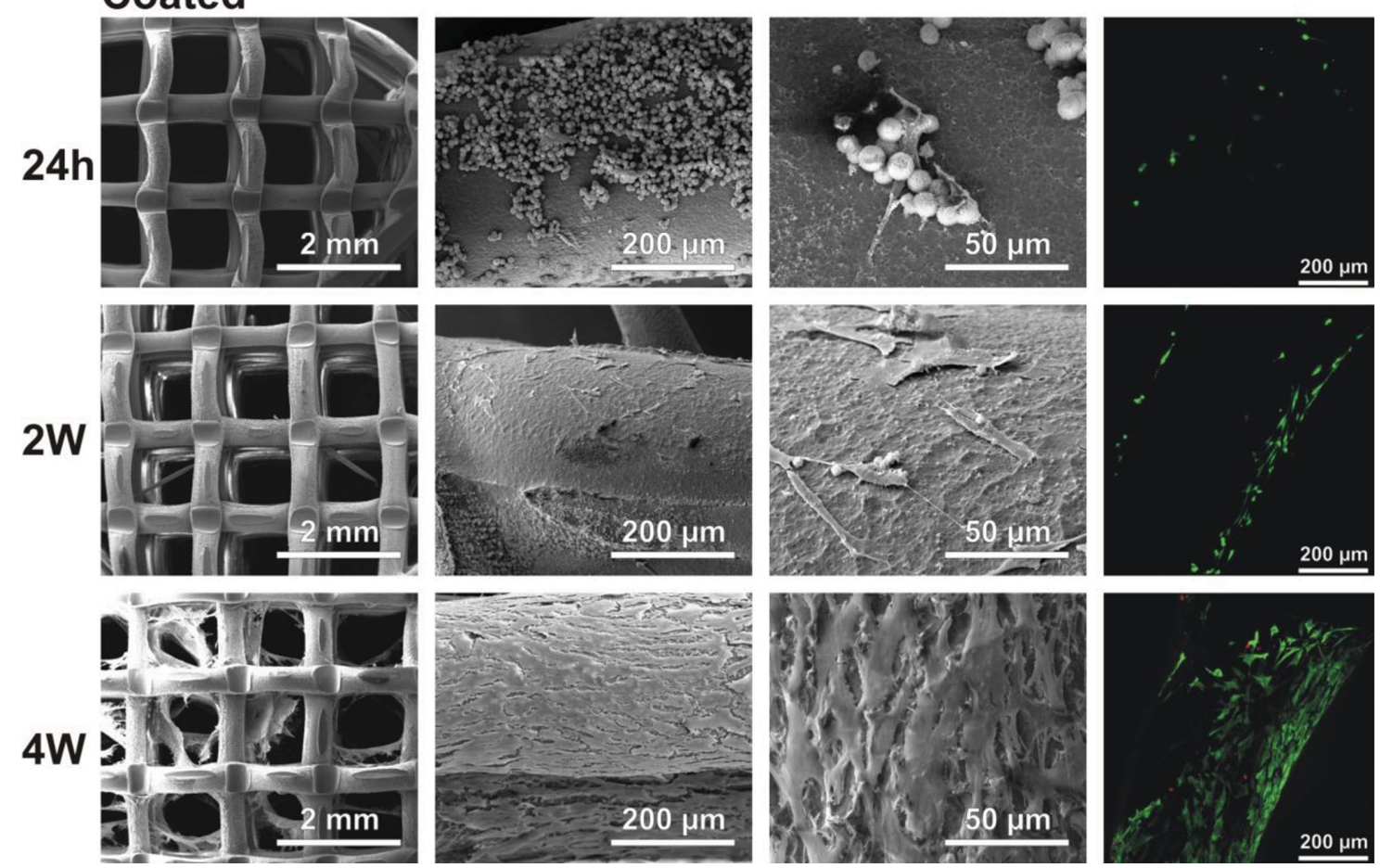

Figure 6. SEM and confocal microscopy (right column) micrographs of noncoated and CaP-coated scaffolds seeded with osteoblasts after 24 h, 2 and 4 weeks of culture. Samples observed through confocal microscopy were stained with FDA/PI showing live cells in green and dead cells in red.

a spacing of $1.5 \mathrm{~mm}$. On the contrary, the volume corresponding to the outer shell device was sliced and converted to G-Code files by using the open source software ReplicatorG (Online ReplicatorC Community). The conversion was performed using two shells, a slice thickness of $0.27 \mathrm{~mm}$, an object infill of $100 \%$, a feed rate of $20 \mathrm{~mm} \mathrm{~s}^{-1}$, and a travel feed rate of $55 \mathrm{~mm} \mathrm{~s}^{-1}$ as parameters. The G-Codes generated through RepRap and ReplicatorG were then merged together in order to generate one single G-Code file. The prototyping of the devices was performed using an open source dual extrusion rapid prototyping machine (Replicator, Makerbot Industries, USA). The materials used were 
Table 1. Statistical analysis of percentage of live cellular content in CaPcoated and noncoated constructs after 24 h, 2 and 4 weeks of culture. Values are not statistically different $(p>0.05)$ showing that the percentage of live cellular content is kept constantly high throughout the 4 weeks culture period in both conditions.

\begin{tabular}{lcc}
\hline Culture period & $\begin{array}{c}\text { Noncoated } \\
{[\%]}\end{array}$ & $\begin{array}{c}\text { Coated } \\
{[\%]}\end{array}$ \\
\hline $24 \mathrm{~h}$ & $97.53 \pm 4.28$ & $93.64 \pm 9.88$ \\
2 weeks & $95.44 \pm 6.34$ & $96.80 \pm 2.00$ \\
4 weeks & $85.67 \pm 8.22$ & $94.73 \pm 3.69$ \\
\hline
\end{tabular}

poly (lactic) acid (PLA) Ingeo 4043D (NatureWorks LLC, USA) for the porous scaffolds and acrylonitrile butadiene styrene (ABS) (Makerbot industries, USA) for the bioreactor chamber. The temperature used for fusing both materials in their corresponding nozzles was $220^{\circ} \mathrm{C}$. Both materials were deposited through coordinated and alternated operation of the nozzles.

Bioreactor Surface Treatment: In order to ensure that fluid leakage could not occur during subsequent cell culture, a posttreatment coating of ABS was applied to the outer bioreactor wall surfaces to close any remaining porosity. In brief, after capping all inlets/outlets, the devices were immersed for $2 \mathrm{~s}$ in an ABS/acetone $60 \mathrm{mg} \mathrm{mL}^{-1}$ solution and then air-dried at room temperature for $30 \mathrm{~min}$. This procedure was performed twice. Finally, the devices were washed in distilled water to remove residual solvent.

Scaffold Surface Treatment: Given the hydrophobic nature of PLA, which could hinder appropriate and homogeneous fluid perfusion through the device, alkaline etching was performed using a $2 \mathrm{M} \mathrm{NaOH}$ solution. The devices were rinsed in $100 \%$ ethanol under vacuum for $1 \mathrm{~h}$ to prewet the scaffold fiber surfaces. After the removal of ethanol, $\mathrm{NaOH}$ solution was perfused through the device until the bioreactor was entirely filled. The devices were incubated for $30 \mathrm{~min}$ at room temperature under vacuum prior to a secondary incubation at $37{ }^{\circ} \mathrm{C}$ for $60 \mathrm{~min}$. Finally, the devices were washed several times with distilled water and then air-dried prior to proceeding to the deposition of a calcium phosphate biomimetic coating on the PLA filaments.

Biomimetic Coating of Scaffolds: The deposition of the calcium phosphate coating was performed by filling the chambers with a total of $8 \mathrm{~mL}$ of $10 \times \mathrm{SBF}$ solution at $\mathrm{pH} 6$ (solution preparation described elsewhere ${ }^{[18,21]}$ ) for $30 \mathrm{~min}$ at $37^{\circ} \mathrm{C}$ and followed by perfusion with a $0.5 \mathrm{M} \mathrm{NaOH}$ solution for $30 \mathrm{~min}$ at $37^{\circ} \mathrm{C}$. Finally, the coated scaffolds were rinsed $5 \times$ with ultrapure water and air-dried.

Microcomputerized Tomography Analysis (MicroCT): The devices were analyzed by MicroCT ( $\mu$ CT40, SCANCO Medical AG, Brüttisellen, Switzerland) at a resolution of $12 \mu \mathrm{m}$, a voltage of $55 \mathrm{kVp}$ and at a current of $175 \mu \mathrm{A}$. 3D images were reconstructed from the scans by the microCT system software.

In Vitro Study: The cell-seeded scaffolds were cultured for up to 4 weeks under bidirectional perfusion. To this end, a $1 \mathrm{~mL}$ sterile syringe was connected to each device's medium inlet using sterile silicone tubing. This apparatus was mounted on a multisyringe adapter placed on an Aladdin syringe pump (World Precision Instruments, USA). A total of 12 devices (each with a dedicated syringe) were simultaneously cultured in this system and therefore 96 scaffolds were cultured at one time. The devices were first sterilized by perfusion with $50 \%$ ethanol solution for $20 \mathrm{~min}$ and dried in a sterile hood for $2 \mathrm{~h}$. Each device was then manually filled with a total of $8 \mathrm{~mL}$ of basal medium and bidirectionally perfused overnight at a flow rate of $0.4 \mathrm{~mL} \mathrm{~min}{ }^{-1}$ with flow inversion occurring every $100 \mathrm{~s}$. This hydration step aimed to promote the preadsorption of proteins onto the scaffold and hence, facilitate subsequent cell attachment. In order to ensure the sterility of the device during the culture period while still allowing gas exchange with the incubator's environment, $0.2 \mu \mathrm{m}$ pore size filters $(13-\mathrm{mm}$ diameter) (Pall, USA) were connected to the outlets positioned at the top of the devices. Primary human osteoblasts were isolated from femur heads of patients undergoing hip surgery. Ethics approval for the use of human osteoblasts in this experiment was granted from the Prince Charles Hospital Ethics Committee (Ethics Clearance No.: EC2310) and Queensland University of Technology ethics committee (Ethics Clearance No.: 0600000232). The osteoblasts were expanded in basal alpha MEM-modified medium (Gibco, USA) supplemented with $10 \%$ fetal calf serum and $1 \%$ penicillin/streptomycin and used at passage 2 . When reaching confluence, the cells were trypsinized and $250 \mu \mathrm{L}$ cell suspensions containing $1 \times 10^{6}$ osteoblasts were injected into the devices through the silicone tube connecting the devices. Since the minifluidic channel network diverted the fluid into eight individual chambers, each scaffold was theoretically seeded with 125000 cells. The devices were bidirectionally perfused for $24 \mathrm{~h}$ at a flow rate of $0.4 \mathrm{~mL} \mathrm{~min}-1$ (resulting in a $0.05 \mathrm{~mL} \mathrm{~min}^{-1}$ flow rate per chamber) in order to allow cell attachment. The perfusion direction was automatically inverted every $100 \mathrm{~s}$ utilizing an external microcontroller connected to the syringe pump. After $24 \mathrm{~h}$, the culture medium was replaced with fresh medium and the flow rate was increased to $0.8 \mathrm{~mL} \mathrm{~min}{ }^{-1}(0.1 \mathrm{~mL} \mathrm{~min}-1$ per chamber), whereas the flow inversion frequency was reduced to $50 \mathrm{~s}$. The medium was changed weekly and the scaffolds were collected for further analysis at $24 \mathrm{~h}, 2$ weeks and 4 weeks postseeding. The scaffold collection was performed as follows: the devices were detached from the syringe pump by removing the silicone tubing and placed in a sterile biohazard safety cabinet. The scaffolds were retrieved from the interior of the bioreactor chambers by manually sectioning the bioreactor walls using scissors and tweezers. The collected scaffolds were processed for DNA content quantification, MTT staining, live/dead assay, confocal laser, and scanning electron microscopy examination.

DNA Content Quantification: For cellular DNA content analysis, the cell-scaffold constructs were frozen at $-80^{\circ} \mathrm{C}$ for at least $48 \mathrm{~h}$. The cell membrane and the extracellular matrix were disrupted in $300 \mu \mathrm{L}$ of $0.5 \mathrm{mg} \mathrm{mL}^{-1}$ Proteinase $\mathrm{K}$ in phosphate buffered EDTA (PBE) at $37{ }^{\circ} \mathrm{C}$ overnight and then transferred into $1.5 \mathrm{~mL}$ eppendorf tubes and further incubated for $24 \mathrm{~h}$ at $60{ }^{\circ} \mathrm{C} .100 \mu \mathrm{L}$ of the diluted $(1 / 50$ in PBE) lysate were aliquoted into black 96 -well plates with $100 \mu \mathrm{L}$ of PicoGreen (P11496, Invitrogen) working solution according to the manufacturer's instructions. After 5 min incubation in the dark, the fluorescence (excitation $485 \mathrm{~nm}$, emission $520 \mathrm{~nm}$ ) was measured using a fluorescence plate reader. A standard curve was also constructed using known concentrations of $\lambda$ DNA provided with the kit. The standards ranged from $10 \mathrm{ng} \mathrm{mL}^{-1}$ to $1 \mu \mathrm{g} \mathrm{mL}^{-1} \lambda \mathrm{DNA}$ and were used to calculate the final DNA content of the sample.

Metabolic Activity Staining: Metabolically, active cells were visualized by using a $1 \mathrm{mg} \mathrm{mL}^{-1}$ MTT solution. Following retrieval, the scaffolds were washed in PBS and then immersed into MTT solution for $30 \mathrm{~min}$ Images were captured by a digital camera mounted on an Eclipse TS100 microscope (Nikon, Tokyo, Japan).

Live/Dead Assay (FDA/PI): Cell viability was assessed using a live/dead assay. For this purpose, the cell-scaffold constructs were washed twice in PBS, incubated for $5 \mathrm{~min}$ at $37^{\circ} \mathrm{C}$ in PBS containing $0.67 \mu \mathrm{g} \mathrm{mL} \mathrm{m}^{-1}$ fluorescein diacetate (FDA) and $5 \mu \mathrm{g} \mathrm{mL}^{-1}$ propidium iodide (PI) (both Invitrogen) and washed again in PBS. The cellularized scaffolds were then analyzed using a confocal laser scanning microscope (Leica TCS SP5, Leica Microsystems, $\mathrm{GmbH}$ ). The excitation/emission wavelengths used for imaging the FDA and PI stainings were 488/518568 and $561 / 598-795 \mathrm{~nm}$, respectively. A semiquantitative analysis of the cellular viability was performed using Image) software to quantify living cells (green color) and dead cells (red color). At least two images from different areas (top and bottom) of the constructs were captured and analyzed for each condition/timepoint.

Scanning Electron Microscopy: Osteoblast morphology and distribution in the scaffolds was assessed at $24 \mathrm{~h}, 2$ and 4 weeks postseeding by scanning electron microscopy. In brief, samples were fixed in $3 \%$ glutaraldehyde, washed in $0.1 \mathrm{M}$ cacodylate buffer, postfixed in $1 \%$ osmium tetroxide in cacodylate buffer for $1 \mathrm{~h}$, prior to dehydration through sequential graded series of ethanol concentrations. Finally, samples were immersed in hexamethyldisilazane for $60 \mathrm{~min}$, air dried, mounted onto aluminum stubs, and gold coated. Samples were 
observed on a FEI Quanta 200 Environmental SEM operating at $10 \mathrm{kV}$. Scaffolds without cells were gold coated prior to imaging.

\section{Supporting Information}

Supporting Information is available from the Wiley Online Library or from the author.

\section{Acknowledgements}

P.F.C. and D.W.H. contributed equally to this work. This work was supported by the Australian Research Council. P.F.C. acknowledges the Portuguese Foundation for Science and Technology for his PhD grant (SFRH/BD/62452/2009).

Received: September 25, 2014 Revised: January 8, 2015 Published online:

[1] R. Langer, J. P. Vacanti, Science 1993, 260, 920.

[2] a) D. W. Hutmacher, J. Biomater. Sci., Polym. Ed. 2001, 12, 107; b) I. Martin, D. Wendt, M. Heberer, Trends Biotechnol. 2004, 22, 80.

[3] D. A. Pereira, J. A. Williams, Br. J. Pharmacol. 2007, 152, 53.

[4] a) C. G. Simon Jr., J. S. Stephens, S. M. Dorsey, M. L. Becker, Rev. Sci. Instrum. 2007, 78, 072207; b) P. Zapata, J. Su, A. J. Garcia, J. C. Meredith, Biomacromolecules 2007, 8, 1907; c) M. Nakajima, T. Ishimuro, K. Kato, I. K. Ko, I. Hirata, Y. Arima, H. Iwata, Biomaterials 2007, 28, 1048; d) D. R. Albrecht, V. L. Tsang, R. L. Sah, S. N. Bhatia, Lab Chip 2005, 5, 111; e) C. G. Simon Jr., N. Eidelman, S. B. Kennedy, A. Sehgal, C. A. Khatri, N. R. Washburn, Biomaterials 2005, 26, 6906; f) J. Kohn, Nat. Mater. 2004, 3, 745; g) D. G. Anderson, S. Levenberg, R. Langer, Nat. Biotechnol. 2004, 22, 863.

[5] a) E. Cukierman, R. Pankov, D. R. Stevens, K. M. Yamada, Science 2001, 294, 1708; b) T. Sun, S. Jackson, J. W. Haycock, S. MacNeil, J. Biotechnol. 2006, 122, 372; c) A. Birgersdotter, R. Sandberg, I. Ernberg, Semin. Cancer Biol. 2005, 15, 405; d) D. W. Hutmacher, Nat. Mater. 2010, 9, 90.

[6] a) A. Goncalves, P. Costa, M. T. Rodrigues, I. R. Dias, R. L. Reis, M. E. Gomes, Acta Biomater. 2011, 7, 1644; b) G. N. Bancroft, V. I. Sikavitsast, J. van den Dolder, T. L. Sheffield, C. G. Ambrose, J. A. Jansen, A. G. Mikos, Proc. Natl. Acad. Sci. U.S.A. 2002, 99, 12600; c) S. H. Cartmell, B. D. Porter, A. J. Garcia, R. E. Guldberg, Tissue Eng. 2003, 9, 1197; d) A. S. Goldstein, T. M. Juarez, C. D. Helmke, M. C. Gustin, A. G. Mikos, Biomaterials 2001, 22, 1279; e) W. L. Grayson, S. Bhumiratana, C. Cannizzaro, P. H. G. Chao, D. P. Lennon, A. I. Caplan, G. Vunjak-Novakovic, Tissue Eng. Part A 2008, 14, 1809; f) Y. F. Han, S. C. Cowin, M. B. Schaffler, S. Weinbaum, Proc. Natl. Acad. Sci. U.S.A. 2004, 101, 16689; g) D. Marolt, A. Augst, L. E. Freed, C. Vepari, R. Fajardo, N. Patel, M. Gray, M. Farley, D. Kaplan, G. Vunjak-Novakovic, Biomaterials 2006, 27, 6138; h) L. Meinel, V. Karageorgiou,
www.MaterialsViews.com

R. Fajardo, B. Snyder, V. Shinde-Patil, L. Zichner, D. Kaplan, R. Langer, G. Vunjak-Novakovic, Ann. Biomed. Eng. 2004, 32, 112; i) I. Martin, T. Smith, D. Wendt, Trends Biotechnol. 2009, 27, 495; j) V. I. Sikavitsas, G. N. Bancroft, A. G. Mikos, J. Biomed. Mater. Res. 2002, 62, 136; k) V. I. Sikavitsas, G. N. Bancroft, H. L. Holtorf, J. A. Jansen, A. G. Mikos, Proc. Natl. Acad. Sci. U.S.A. 2003, 100, 14683; I) J. van den Dolder, G. N. Bancroft, V. I. Sikavitsas, P. H. M. Spauwen, J. A. Jansen, A. G. Mikos, J. Biomed. Mater. Res. A 2003, 64A, 235; m) M. Frohlich, W. L. Grayson, D. Marolt, J. M. Gimble, N. Kregar-Velikonja, G. Vunjak-Novakovic, Tissue Eng. Part A 2010, 16, 179.

[7] a) S. J. Hollister, Nat. Mater. 2005, 4, 518; b) F. P. W. Melchels, M. A. N. Domingos, T. J. Klein, J. Malda, P. J. Bartolo, D. W. Hutmacher, Prog. Polym. Sci. 2012, 37, 1079; c) D. W. Hutmacher, S. Cool, J. Cell. Mol. Med. 2007, 11, 654; d) D. W. Hutmacher, M. Sittinger, M. V. Risbud, Trends Biotechnol. 2004, 22, 354; e) R. Grylls, Machine Design 2003, 75, 56.

[8] P. F. Costa, C. Vaquette, J. Baldwin, M. Chhaya, M. E. Gomes, R. L. Reis, C. Theodoropoulos, D. W. Hutmacher, Biofabrication 2014, 6, 035006

[9] a) H. V. Unadkat, M. Hulsman, K. Cornelissen, B. J. Papenburg, R. K. Truckenmuller, G. F. Post, M. Uetz, M. J. T. Reinders, D. Stamatialis, C. A. van Blitterswijk, J. de Boer, Proc. Natl. Acad. Sci. U.S.A. 2011, 108, 16565; b) M. P. Lutolf, R. Doyonnas, K. Havenstrite, K. Koleckar, H. M. Blau, Integr. Biol. 2009, 1, 59; c) Y. Mei, Mater. Today 2012, 15, 444.

[10] a) M. J. Jaasma, N. A. Plunkett, F. J. O'Brien, J. Biotechnol. 2008, 133, 490; b) F. W. Janssen, J. Oostra, A. van Oorschot, C. A. van Blitterswijk, Biomaterials 2006, 27, 315

[11] L. A. Hidalgo-Bastida, S. Thirunavukkarasu, S. Griffiths, S. H. Cartmell, S. Naire, Biotechnol. Bioeng. 2012, 109, 1095.

[12] J. F. Alvarez-Barreto, S. M. Linehan, R. L. Shambaugh, V. I. Sikavitsas, Ann. Biomed. Eng. 2007, 35, 429.

[13] X. Tian, S. Wang, Z. Zhang, D. Lv, PLoS One 2012, 7, e42804.

[14] a) E. Figallo, C. Cannizzaro, S. Gerecht, J. A. Burdick, R. Langer, N. Elvassore, G. Vunjak-Novakovic, Lab Chip 2007, 7, 710; b) M. Y. Rotenberg, E. Ruvinov, A. Armoza, S. Cohen, Lab Chip 2012, 12, 2696.

[15] E. Gottwald, S. Giselbrecht, C. Augspurger, B. Lahni, N. Dambrowsky, R. Truckenmuller, V. Piotter, T. Gietzelt, O. Wendt, W. Pfleging, A. Welle, A. Rolletschek, A. M. Wobus, K. F. Weibezahn, Lab Chip 2007, 7, 777.

[16] a) J. Yang, Y. Q. Wan, C. F. Tu, Q. Cai, J. Z. Bei, S. G. Wang, Polym. Int. 2003, 52, 1892; b) H. Tsuji, T. Ishida, N. Fukuda, Polym. Int. 2003, 52, 843 .

[17] A. L. Oliveira, S. A. Costa, R. A. Sousa, R. L. Reis, Acta Biomater. 2009, 5, 1626.

[18] C. Vaquette, S. Ivanovski, S. M. Hamlet, D. W. Hutmacher, Biomaterials 2013, 34, 5538.

[19] a) M. T. Arafat, C. X. F. Lam, A. K. Ekaputra, S. Y. Wong, X. Li, I. Gibson, Acta Biomater. 2011, 7, 809; b) J. V. Araujo, A. Martins, I. B. Leonor, E. D. Pinho, R. L. Reis, N. M. Neves, J. Biomater. Sci., Polym. Ed. 2008, 19, 1261

[20] P. F. Costa, C. Vaquette, Q. Zhang, R. L. Reis, S. Ivanovski, D. W. Hutmacher, J. Clin. Periodontol. 2014, 41, 283.

[21] F. Yang, J. G. C. Wolke, J. A. Jansen, Chem. Eng. J. 2008, 137, 154 